\title{
GEOLOCALIZACIÓN DE INFORMACIÓN Y MAPEO DE DATOS EN PERIODISMO ONLINE CON USHAHIDI
}

\section{Geolocation of information and data mapping with Ushahidi in online journalism}

Teresa Sandoval-Martín y Óscar Espiritusanto

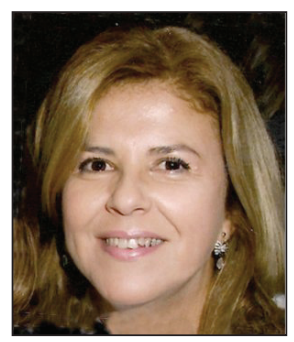

Teresa Sandoval-Martín es profesora titular de periodismo en la Universidad Carlos III de Madrid. Doctora europea y premio extraordinario de tesis doctoral. Primera promoción del Master universitario en nuevas tecnologías de la información y de la comunicación de la UNED. Coautora de diversos libros de periodismo e internet, ha publicado numerosos artículos sobre este tema. Es miembro de la junta directiva de la Sociedad Española de Periodística (SEP).

http://orcid.org/0000-0001-9220-2299

msandova@hum.uc3m.es

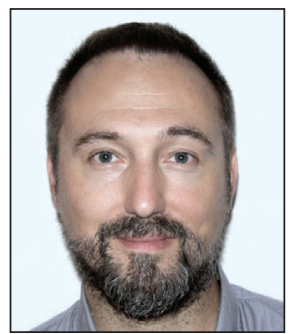

Óscar Espiritusanto es fundador de PeriodismoCiudadano.com, observatorio para la promoción y fomento de iniciativas de medios colaborativos. Profesor asociado de periodismo en la Universidad Carlos III de Madrid, imparte cursos de postgrado en diversas empresas e instituciones. Coautor del libro Periodismo ciudadano: Evolución positiva de la comunicación y de informes anuales sobre el uso de las tecnologías.

http://orcid.org/0000-0003-2285-9265

oespirit@hum.uc3m.es

Universidad Carlos III de Madrid, Departamento de Periodismo y Comunicación Audiovisual C/ Madrid 126. 28903 Getafe (Madrid), España

\section{Resumen}

Las experiencias de crowdsourcing y crowdmapping con herramientas como Ushahidi (testimonio o testigo en swahili) permiten a individuos, instituciones y organizaciones periodísticas aprovechar el flujo informativo de los usuarios, aportando valor a la cadena comunicativa, aunque el proceso no está exento de algunas dificultades. Esta plataforma ofrece desde 2008 varias aplicaciones de software libre. Primero se centró en representar datos a través de un mapa participativo de información geolocalizada y luego en la verificación de la información aportada por los ciudadanos. A partir de la bibliografía y hemerografía existente sobre esta temática y de los proyectos desarrollados, se muestra un área específica de colaboración entre los periodistas profesionales y los periodistas ciudadanos en la creación, distribución y verificación de contenidos. Los medios internacionales que han aplicado Ushahidi son: The guardian, BBC, Al jazeera y Huffington post.

\section{Palabras clave}

Geolocalización de información; Geolocalización de datos; Periodismo digital; Medios digitales; Periodismo ciudadano; Crowdsourcing; Crowdmapping; Periodismo de datos; Mapas; Generación de contenido por el usuario; Curación de contenidos; Ushahidi; Crowdmap.

\footnotetext{
Abstract

Crowdsourcing and crowdmapping tools such Ushahidi (testimony or witness in Swahili) give value to the communication chain. They allow individuals, institutions, and journalistic organizations to take advantage of the flow of information provided by the users, although the process is not without some difficulties. Ushahidi, based on free software, offers a broad range of applications to be used in media contexts. First, Ushahidi focuses on data through a participatory map of georeferenced information and then it addresses the verification of the information provided by the citizens. From the analysis of the bibliography and some study cases, we describe a specific area of collaboration between professional journalists and citizen journalists in the creation, distribution, and verification of content. International media which have used Ushahidi include The guardian, BBC, Al jazeera and Huffington post.
} 


\section{Keywords}

Geolocation; Data geolocation; Online journalism; Online media; Citizen journalism; Crowdsourcing; Crowdmapping; Data journalism; Maps; User generated content; Content curation; Ushahidi; Crowdmap.

Sandoval-Martín, Teresa; Espiritusanto, Óscar (2016). "Geolocalización de información y mapeo de datos en periodismo online con Ushahidi". El profesional de la información, v. 25, n. 3, pp. 458-472.

http://dx.doi.org/10.3145/epi.2016.may.16

\section{Introducción ${ }^{1}$}

La actual coyuntura tecnológica favorece el afianzamiento del llamado "quinto poder" en relación con la capacidad de participación de los ciudadanos a través de internet en numerosos procesos, como es el caso de las reformas legislativas. En este contexto global de open government y open source se están consolidando fenómenos como el del periodismo ciudadano o participativo (Domingo et al., 2008, García-de-Torres, 2010; Singer et al., 2011; Espiritusanto; Gonzalo-Rodríguez, 2011; Allan, 2013; Holton; Coddington; Gil-de-Zúñiga, 2013; Mortensen; Keshelahvili; Weir, 2016) y el crowdsourcing (Howe, 2006; 2009; Brabham, 2008; 2013; Estellés-Arolas; GonzálezLadrón-De-Guevara, 2012a; 2012b). Ello ha favorecido la aplicación del crowdsourcing en los medios de comunicación así como el inicio de investigaciones sobre crowdsourcing journalism (Aitamurto, 2015, 2016), entre las que se inscribe este estudio exploratorio sobre su uso mediante la aplicación de geolocalización Ushahidi.

La geolocalización permite conocer, analizar y divulgar datos antes imposibles de recopilar

Para los autores más referenciados en este campo (Gillmor, 2004; Rheingold, 2004; Howe, 2009; Shirky, 2008), se trata del nuevo poder de las masas, de grupos de ciudadanos o de multitudes inteligentes que aportan valor, conocimiento o recursos a determinados proyectos, eventos o instituciones. Entre estas aportaciones se ha sumado en los últimos años la posibilidad de ubicar geográficamente en mapas en tiempo real información enviada por quienes participan en un determinado proyecto. Esto ha sido posible gracias a plataformas como Ushahidi (que significa testimonio o testigo en swahili), que permite conocer, analizar y divulgar datos antes imposibles de recopilar de forma sincrónica.

https://www.ushahidi.com

La participación activa de numerosas personas en la generación de contenido a través de SMS, mail, Twitter y las actuales posibilidades tecnológicas favorecen el auge de sistemas de geolocalización de información móvil en mapas (Gonzalo-Rodríguez, 2011). Además de Ushahidi destacan Google maps y OpenStreetMap (OSM, convertida en un equivalente a la Wikipedia de los mapas), entre las más consolidadas.

Las aplicaciones de geolocalización, al indicar la posición de una persona u objeto en el espacio, gracias a internet, enlazan lo local y lo global a través de la nube, como destaca Beltrán (2016).
Se han convertido en indispensables para ONGs internacionales y autoridades locales en casos como las crisis humanitarias, una vez demostrada su eficacia en el terremoto de Haití de 2010. A partir de entonces Ushahidi ha tenido un destacado papel en la creación de información interactiva en situaciones de crisis específicas en las que las organizaciones humanitarias y los medios de comunicación tradicionales se encuentran con grandes dificultades de acceso al terreno. Desde su creación en 2007 esta herramienta ha evolucionado y demostrado su valor en numerosas ocasiones (figura 1) y "es reconocida como una de las organizaciones que lidera el ámbito del crowdsourcing" (Greengard, 2011, p. 21)

OpenStreetMap ha creado también un proyecto específico en este ámbito, Humanitarian OpenStreetMap Team (HOT) (Plaza, 2014), con el fin de colaborar en situaciones humanitarias de emergencia, una tendencia global si nos fijamos en el aumento de organizaciones similares enfocadas a la gestión de crisis (Gonzalo-Rodríguez, 2016). No es el único campo en que resultan útiles los mapas realizados en colaboración con los ciudadanos, y sus posibilidades parecen infinitas (Internews Center for Innovating \& Learning, 2012). No obstante, en los medios de comunicación su aplicación está todavía en un estado incipiente, aunque las experiencias halladas muestran sus posibilidades. El crowdsourcing aplicado al periodismo es una tendencia en las agendas investigadoras de centros universitarios de prestigio, como el Tow Center for Digital Journalism de la Columbia Journalism School de Nueva York y el Brown Institute for Media Innovation de la Stanford University.

\section{Ushahidi tiene un papel destacado cuan- do los periodistas no pueden acceder al terreno}

En el caso de Ushahidi se trata de una colaboración online mediante software libre y de fácil uso, que permite la validación de las informaciones recibidas. La información con el qué, el cuándo y el dónde, queda disponible para ciudadanos, gobiernos, instituciones y medios para su posterior utilización y análisis. La aplicación permite agregar noticias aparecidas en los medios sobre el tema tratado.

En 2012 en su página web apareció por primera vez el término citizen journalism. Su apuesta por el periodismo ciudadano (Ushahidi, 2012), la convierte en una plataforma con un importante potencial para la colaboración entre medios y ciudadanos, como se mostrará en varios ejemplos. 


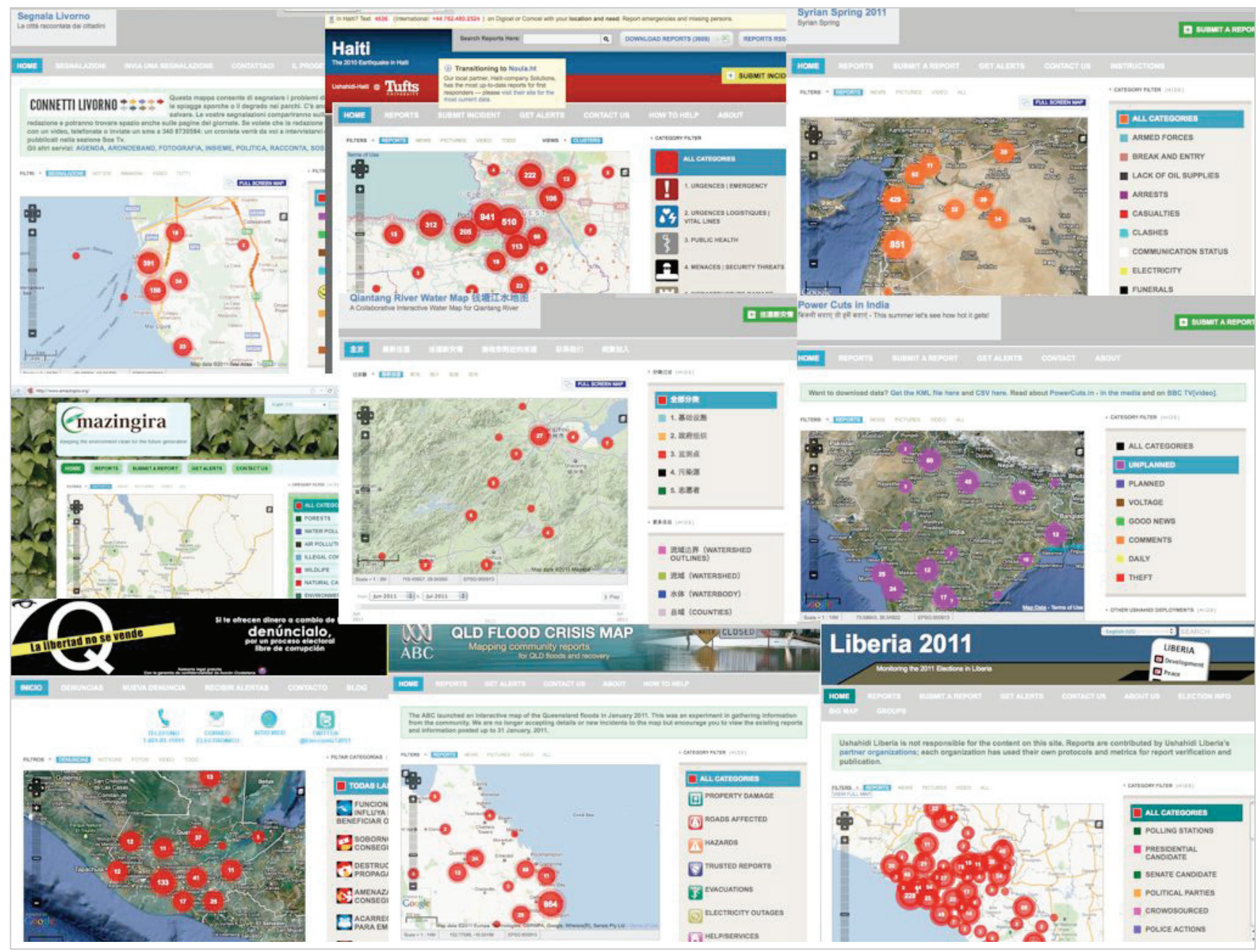

Figura 1. Ejemplos de uso de Ushahidi

https://wiki.ushahidi.com/display/WIKI/Deployments+of+the+Week

La directiva de Ushahidi está compuesta por Erik Hersman (director-cofundador), David Kobia (cofundador), Juliana Rotich (cofundadora), Clay Shirky, Nora Denzel y Sally Chin (Ushahidi, 2015a). En los últimos años la fundación ha recibido varios premios: The MacArthur Award (2013) y Global Adaptation Index Prize (2012). En 2010 fue reconocida su valía por el Foro Económico Mundial (Gonzalo-Rodríguez, 2011).

Esta organización forma parte de un movimiento global, de transformación integral de numerosos sectores. Como señala Rheingold (2011) en el prólogo de Periodismo ciudadano. Evolución positiva de la comunicación (Espiritusanto; Gonzalo-Rodríguez, 2011, p. VII):

"Ios medios digitales, en manos de miles de millones de personas, están cambiando las instituciones y la práctica profesional del periodismo y, por tanto, también la propia naturaleza de la democracia".

Peter Lee, director de uno de los departamentos tecnológicos de la agencia de defensa de los Estados Unidos, Darpa, cree que el crowdsourcing:

"está modificando la forma en que los gobiernos, las instituciones y otros abordan situaciones complejas y problemas" (Greengard, 2011, p. 22).
Este artículo se estructura en los siguientes apartados:

- objetivo y justificación de este trabajo;

- oarco teórico y conceptos clave, fruto de una revisión exhaustiva de las publicaciones científicas sobre crowdsourcing y periodismo, crowdmapping y Ushahidi;

- características de Ushahidi, primeros proyectos que tuvieron un gran impacto, así como otros ejemplos de interés para este estudio. Se incluyen las referencias acerca de los problemas que conlleva la verificación y la protección de los ciudadanos reporteros;

- resultados de la investigación acerca de los medios de comunicación que han utilizado la aplicación, seguida de las conclusiones, que incluyen las limitaciones y carencias de la investigación.

\section{Explorar el crowdmapping en los medios}

Aunque cada vez son más las historias en las que se solicita la ayuda de los usuarios (para informarse a través de fuentes directas, conocer impresiones o colaborar en la escritura de una historia), existen lagunas importantes en la investigación sobre la repercusión de estas prácticas en el periodismo. Aitamurto (2016) considera que estas carencias se hacen más latentes en cuanto al conocimiento de lo que aporta esta práctica, enmarcada en lo que denomina 
open journalism. Si bien los trabajos de esta investigadora y directora del Brown Institute for Media Innovation de la Stanford University sobre varios casos desarrollados con su ayuda en Finlandia, arrojan luz sobre este fenómeno, es necesario ampliar los enfoques, profundizar en las líneas ya abiertas e incluirlo en la agenda de la investigación científica en periodismo.

Desde el ámbito académico y docente del periodismo destacan los esfuerzos realizados por el Tow Center for Digital Journalism de la Columbia University, al elaborar una guía de crowdsourcing (Onuoha; Pinder; Schaffer, 2015) mediante el estudio de casos relacionados con sus usos periodísticos.

\section{Los ciudadanos vigilan cada vez más los procesos electorales con herramientas tecnológicas}

Destacan también las aportaciones de Bradshaw y Brightwell (2012) en el ámbito del periodismo de investigación y la búsqueda de fuentes entre los ciudadanos.

Brabham (2008; 2013) es conocido por haber realizado sendos estudios sobre las aplicaciones del crowdsourcing en varios ámbitos, y Estellés-Arolas y González-Ladrón-DeGuevara (2012a; 2012b) por sus trabajos en busca de una definición para este término.

El objetivo principal de este estudio era explorar desde la perspectiva de la periodística (disciplina que estudia la práctica profesional) (López-Pan, 2010), las posibilidades que brinda la submodalidad del crowdsourcing llamada crowdmapping al periodismo, a través de las experiencias de uso de Ushahidi en medios, abriendo de esta manera nuevas vías para la investigación en el campo del mapeo de información de valor noticioso combinándolo con el crowdsourcing. Tratamos con ello de responder a algunas preguntas:

- ¿quién está realizando crowdmapping en medios?

- ¿con qué finalidad?

- ¿qué ventajas e inconvenientes conlleva su uso?

Dada la dificultad que encierra la búsqueda de materiales en los medios digitales, debido a la pérdida de los trabajos periodísticos que no se archivan en las hemerotecas digitales y debido a que Ushahidi es la plataforma de más arraigo en el ámbito del crowdmapping, se decidió centrar el objeto de estudio en esta herramienta.

Mediante la búsqueda sistemática en bases de datos científicas y de medios de comunicación, la lectura de documentos elaborados por Ushahidi u otras organizaciones y los resultados en buscadores relacionados con la creación de mapas con Ushahidi, en especial por parte de medios de comunicación, se pudieron localizar y analizar los experimentos llevados a cabo en este ámbito concreto. Al no existir investigaciones previas, los resultados obtenidos aunque conforman una pequeña muestra, arrojan luz sobre el uso de Ushahidi en los medios.

Los ejemplos sobre su uso por grandes medios de comunicación internacionales que se localizaron fueron los realizados por BBC, The guardian, Al jazeera y The Huffington post. También se encontraron ejemplos en medios locales o hiperlocales como Houston chronicle, Watershed post, la emisora de radio pública WNYC y el portal Hamilton reporter, que también se han tenido en cuenta. A pesar de que se ha realizado una búsqueda exhaustiva, no se puede descartar que se hayan podido realizar otros mapas con Crowdmap de Ushahidi tanto en medios locales como nacionales e internacionales.

https://hamiltonreporter.crowdmap.com

\section{Marco teórico y conceptos clave}

La primera vez que se utilizó el término crowdsourcing fue en un artículo de Jeff Howe en la revista Wired en junio de 2006. Posteriormente este autor publicó en su libro Crowdsourcing: Why the power of the crowd is driving the future of business (2009) dos definiciones:

“(...) la primera defiende que es el acto de tomar un trabajo realizado tradicionalmente por un agente designado (generalmente un empleado) y la externalización a un grupo indefinido, generalmente grande, de personas en la forma de convocatoria abierta y la segunda, como la aplicación de los principios de código abierto a los campos externos del software".

En el ámbito periodístico interesan estas definiciones de Howe, en esencia, porque para que se lleve a cabo en el marco de una redacción por un equipo de periodistas e informáticos, es necesaria la aplicación de los principios del software libre, por tanto, una herramienta como Ushahidi. Cabe señalar que en este caso no existe una externalización empresarial, ni candidatos preseleccionados sino que la convocatoria se realiza directamente a la crowd (masa). Ésta participa de manera voluntaria, de momento y en los casos estudiados, sin recibir más compensación que el poder acceder a la información que entre todos generan. Una llamada en abierto a la masa a través de internet para generar participación.

Menos académico es el término crowdmapping referido a la aplicación del crowdsourcing sobre mapas. Aunque apenas hay bibliografía que utilice literalmente ese término (crowdmapping) y que lo señale como una subespecialidad del crowdsourcing (Aitamurto, 2015; Furtado et al., 2012; Grove, 2015), abundan las referencias académicas que analizan Ushahidi desde distintos campos, principalmente los de computer science y geografía, así como desde perspectivas más cercanas a los estudios en comunicación y los nuevos medios digitales (Palmer, 2014; Bailard; Livingston, 2014; Brandusescu; Sieber; Jochems, 2015). Uno de los textos más referenciados es el de Patrick Meier (2012), miembro de Ushahidi.

Sobresalen los trabajos que ahondan en uno de los temas más controvertidos de esta práctica: las vulnerabilidades de estos mapas de crisis en zonas de conflicto o donde no se respetan determinados derechos fundamentales. Chamales y Baker $(2011)^{2}$ muestran los peligros que corren los reporteros ciudadanos en regímenes opresivos y por el uso de información no verificada $y$, por último, se menciona un artículo de López-Guillén, Flores-Mendoza y Welti-Santos (2011) que cita un estudio de caso en el que se aplicó Ushahidi en un contexto local -un ámbito con grandes posibilidades-, el tráfico en la ciudad de México. 
Especial mención merece el artículo de Furtado et al. (2012) "Open government and citizen participation in law enforcement via crowd mapping", temas muy en boga en el discurso político de hoy en día, al igual que la transparencia.

En su conjunto, la revisión de la bibliografía científica indica que existe un enorme interés en los últimos años en abordar desde distintos puntos de vista el fenómeno del crowdsourcing, especialmente, en situaciones de crisis en las que los ciudadanos afectados envían información que se geolocaliza en mapas interactivos, la mayor parte de las veces en tiempo real.

De la revisión de la bibliografía en los temas relacionados con las infotecnologías (computer science) podemos concluir que la definición de crowdsourcing ha evolucionado hacia lo que se denomina computación humana (human computation), informática social (social computing) e inteligencia colectiva, como bien distinguen Quinn y Bederson (2011, p. 1404) de la University of Maryland. Estos términos han surgido de la evolución de la relación hombre-máquina en un escenario sociotécnico complejo caracterizado por la popularización de los medios sociales para la información, la relación y la comunicación propios de la web 2.0.

De los conceptos mencionados nos interesa especialmente el de inteligencia colectiva, cuya definición está ampliamente consensuada:

"grandes grupos de personas carentes de organización pueden llevar a cabo grandes cosas trabajando juntos" (Quinn; Bederson, 2012, p. 1405).

\section{Existe un creciente interés por el crowd-} sourcing en situaciones de crisis en las que los ciudadanos envían información geolocalizada en mapas interactivos en tiempo real

La inteligencia colectiva, término acuñado por Pierre Lévy (2004), muestra cómo individuos con ciertas cuotas de conocimiento colaborando entre ellos pueden generar más y mejor conocimiento y así crear una inteligencia que esté por encima (en lo que a conocimiento se refiere) de todo el grupo.

El proceso de co-evolución en el que se encuentran sumidas ciudadanía y tecnología, impacta en profesiones como la periodística, introduciendo corrientes como el periodismo de datos y el periodismo informático o computational journalism (Cohen; Hamilton; Turner, 2011).

La participación ciudadana es otra de las corrientes que se ha convertido en un recurso valioso para el periodismo, que lo transforma drásticamente. Como señala Fumero-Reverón (2011, p. 606), "el fenómeno del periodismo ciudadano se consolida con el tiempo y empieza a calar en numerosos actores tradicionales, cabeceras conocidas y grupos mediáticos".

Paralelamente van surgiendo cada vez más iniciativas que implican la participación de las audiencias en la información.

Los ejemplos más conocidos de crowdsourced journalism incluyen los proyectos de los equipos de investigación de The guardian, como:
- examen de cientos de documentos relacionados con los políticos británicos en 2009, que supuso un escándalo (Aitamurto, 2011; Daniel; Flew, 2010);

- cobertura del huracán Irene en Estados Unidos por periodistas crowdsourcers (Dailey; Startbird, 2014).

Los dos casos son buenos ejemplos de los dos tipos de usos de periodismo ciudadano, crowdsourced journalism u open journalism ${ }^{3}$ en los medios:

- periodismo de investigación,

- situaciones de crisis producidas por un desastre natural en las que no sólo la función social del periodismo debe estar presente sino también el ejercicio del periodismo de servicio (Diezhandino-Nieto, 1994).

\section{Los dos casos más habituales de usos del periodismo ciudadano o crowdsourced journalism son periodismo de investiga- ción y situaciones de crisis}

En estas dos especialidades del periodismo, la segunda requiere inmediatez de reacción y gestión y la primera no. En los casos señalados, los periodistas usaron contenidos generados por los usuarios una vez aplicados los procedimientos de confirmación propios del ejercicio profesional del periodismo.

En las situaciones de crisis y en los ejercicios de vigilancia y control de los procesos electorales y de denuncia de los abusos de poder, así como de los derechos humanos, es donde ha surgido en mayor medida la colaboración entre ciudadanos y periodistas (Espiritusanto; Gonzalo-Rodríguez, 2011). Ello debería reflejarse en la realización de mapas de información geolocalizada, puesto que el mayor calado de Ushahidi tanto entre los creadores de proyectos como por la participación ciudadana, se ha dado también en estos entornos (Internews Center for Innovating \& Learning, 2012).

Mostrar el cuándo, el dónde y el qué tiene sentido:

- en una catástrofe, como son los huracanes y los terremotos (de hecho, pueden encontrarse numerosos ejemplos de Ushahidi en las zonas del Caribe);

- para denunciar o registrar crímenes y abusos de los derechos humanos (con numerosas muestras principalmente en Oriente Medio y Norte de África);

- para visibilizar o denunciar irregularidades en procesos electorales en numerosos países (con una importante cantidad de casos en Latinoamérica y África);

- conflictos armados;

- problemáticas sociales;

- infraestructuras de emergencias.

Aunque los mapas no hayan sido realizados por periodistas sino por comunidades de voluntarios, tratan cuestiones que deberían ser tratados por los medios si quieren cumplir con su función social (Diezhandino-Nieto, 2007). Por ello no es de extrañar que se empiecen a realizar mapas experimentales en los medios en torno a estos temas, como veremos. 
Para Aitamurto (2015, p. 3525), que ha centrado una parte de sus investigaciones en la motivación de los usuarios a participar:

"La participación en el crowdsourcing es a menudo rápida, en un solo acto. El periodismo ciudadano puede utilizar el crowdsourcing en sus informaciones, y los medios digitales pueden promoverlo mediante la formulación de preguntas a los ciudadanos reporteros para que envíen un determinado tipo de noticias (...)" (Aitamurto, 2015).

Como señala esta autora, no todo el periodismo participativo utiliza el crowdsourcing como método para investigar $u$ obtener conocimiento. Se puede usar también para atraer visitantes a la página o para fidelizar al lector y con ello obtener un rendimiento económico a través de los anunciantes. Para esta investigadora de la Stanford University, en el terreno de la investigación en crowdsourced journalism y periodismo participativo, hasta el momento los trabajos se han focalizado en la perspectiva del periodista (Hänska-Ahy; Shapour, 2013; Singer et al., 2011), por lo que aboga por descubrir los motivos que llevan a la masa a colaborar en las propuestas de los medios.

\section{Ventajas y desventajas}

Samuel Greengard (2011, p. 20) califica al crowdsourcing como simple pero poderoso:

"Prácticamente cualquiera tiene el potencial para conectar información valiosa".

Patrick Meier, Director of Crisis Mapping en Ushahidi, comenta a Greengard:

"El crowdsourcing ofrece tanto ventajas como desventajas. (...) Es muy eficiente en la recopilación de información de forma rápida y eficaz. Puede ayudar a generar una velocidad de respuesta en una etapa inicial de un desastre".

Pero las preocupaciones que se generan sobre la exactitud de los datos no son infundadas. Añade que:

"Uno de los mayores retos en el desarrollo de aplicaciones de este tipo es generar fuentes de confianza" (Greengard, 2011, p. 22).

Plataformas como Ushahidi cuentan con herramientas que permiten seleccionar el contenido entrante generado por los usuarios casi en tiempo real.

Los autores citados reflejan algunos de los problemas que rodean a esta práctica y a la herramienta en sí. No obstante, el informe más exhaustivo sobre las experiencias de uso de los creadores y participantes en esos proyectos es Mapping de maps (Internews Center for Innovating \& Learning, 2012).

Otros investigadores han expuesto su preocupación por el uso indebido de terceros de la información geolocalizada de los reporteros ciudadanos en los regímenes opresivos, al igual que por el hecho de que puedan enviarse mensajes intencionados para posteriormente llevar acciones violentas. Determinar la veracidad de la información es complicado debido a que dependiendo del contexto se podrán requerir diferentes niveles de verificación: "las necesidades de verificación de aquellas organizaciones que mapean los abusos cometidos en el ámbito de los derechos humanos son diferentes a las de aquellas organizaciones que necesitan identificar a la población vulnerable para el envío de alimentos" (Chamales; Baker, 2011).

Se están haciendo esfuerzos por elaborar manuales o guías de crowdsourcing en el área humanitaria que incluyen procesos y métodos para verificar las informaciones suministradas por los reporteros ciudadanos, como es el caso de Amnistía Internacional que ha creado Citizen Evidence Lab. https://citizenevidence.org

Desde el entorno académico se ha elaborado una guía de crowdsourcing para periodistas y medios que incluye un apartado específico sobre verificación (Onuoha; Pinder; Schaffer, 2015). Una especial mención en la lucha por visibilizar mediante vídeos los abusos y las violaciones de los derechos humanos en todo el mundo y que llevan años aplicando el crowdsourcing y realizando recomendaciones y guías para los reporteros ciudadanos es Witness, creada en 1992 por el cantante y activista Peter Gabriel.

https://witness.org

En periodismo los requerimientos de verificación deben ser específicos para este campo, y en consonancia, deben producirse cambios en el periodismo, en la línea del open journalism descrito por Aitamurto (2016). Ello conlleva una modificación de las actuales prácticas, más cerradas y constreñidas a los principios clásicos, que sin perderlos deben ser repensados y adaptados a nuevas realidades comunicativas y colaborativas. Son indispensables una mayor profundización y teorización en el terreno de la verificación y la protección de los informantes, así como en todas sus connotaciones para la práctica profesional. Aunque escapan al objetivo principal de este estudio, centrado en los usos que los medios están haciendo de la plataforma Ushahidi, no se descarta su abordaje en un futuro.

\section{Ushahidi}

Ory Okolloh, nacida en Kenia, graduada en la Harvard Law School, directora general y cofundadora de Ushahidi, junto con Juliana Rotich, Erik Hersman y David Kobia, tuvo la idea de poner esta plataforma en marcha al descubrir la escasa información y la censura gubernamental existente en los medios de comunicación tradicionales durante el conflicto post electoral que asoló Kenia en 2008.

Como autora de uno de los blogs keniatas más populares, informó de la terrible situación de su país publicando datos de interés para los ciudadanos y criticó duramente al gobierno por no hacer nada para ayudar a los desplazados. Su información permitió ayudar a un gran número de personas que comenzaron a responder a su petición de información con gran agilidad.

http://www.kenyanpundit.com

Así surgió la idea de crear un mapa interactivo en el que todos los afectados pudieran plasmar información de crisis. Una sencilla plataforma que permitiera publicar informes ciudadanos y geolocalizarlos en un mapa en tiempo real 
a través de mensajes SMS, e-mails o vía Web, así nació Ushahidi. Desde entonces esta aplicación ha gestionado con eficacia algunos de los ingredientes fundamentales del nuevo panorama comunicativo:

- software de código abierto

- información participativa

- nuevas herramientas tecnológicas

- geolocalización.

El equipo de Ushahidi se convirtió en una organización sin ánimo de lucro y creó una aplicación gratuita que pudiera ser utilizada por organizaciones y ciudadanos de todo el mundo. El objetivo principal era crear un sistema de alerta temprana que ayudara en la visualización de datos para una respuesta y recuperación más rápida y eficaz en situaciones de crisis, pero luego los usos se han ido ampliando, en tanto que se puede aplicar a lo que la gente quiera.

En poco tiempo la aplicación creció y se hizo más sencilla. Surgió Crowdmap y con ella la posibilidad de crear un mapa sin tener que instalarlo en un servidor web. Desde entonces ciudadanos y organizaciones disponen de una aplicación de mapeado de información con un enorme potencial.

Una vez demostrada su utilidad, Ushahidi pasó a abordar la verificación de la información, y creó Swiftriver. Nació de la necesidad de comprender y actuar sobre una gigantesca ola de datos en momentos de crisis, que tienden a desbordar a quienes los reciben en las primeras 24 horas, con la consiguiente necesidad de verificarlos de la forma más eficiente y rápida posible. La información en tiempo real se filtra por la masa, se visualiza y organiza. Existen herramientas en el mercado que realizan este tipo de tareas, la mayoría propietarias y de costes elevados. El objetivo de Swiftriver era ofrecer una que fuera fácil de usar y, nuevamente, de código abierto.

En una entrevista realizada por Óscar Espiritusanto (periodismociudadano.com, 2010) al profesor Shirky en 2009, éste afirmaba que los dispositivos móviles son una de las llaves que hacen posible el periodismo ciudadano. Gran parte de la documentación llega hoy en día de forma inmediata a través de los teléfonos móviles de los ciudadanos. Es el útil más importante para el periodismo ciudadano.

Dado que internet puede ser de difícil acceso o no estar disponible en algunas partes del mundo, el equipo de desarrollo de Ushahidi tiene en cuenta una movilidad simple para aquellos que no tienen acceso a las últimas innovaciones en dispositivos de telefonía móvil ni smartphones. Para ello sigue pensando en tecnologías como el SMS para la comunicación entre individuos y plataformas, entre móviles e internet.

El último avance tecnológico de Ushahidi se ha centrado en la conectividad de los ciudadanos en países poco desarrollados. Ory Okolloh confirmó, en una entrevista concedida a The guardian (Davidi; Berliner, 2014) como directora de inversiones de Omidyar Network Africa, que la tecnología está cambiando la diseminación de información en África y está ayudando a amplificar las voces de los ciudadanos. La conexión a internet en diversas partes de África puede verse frustrada por las diferentes señales y un suministro de electricidad intermitente. Para solventar este problema Ushahidi ha creado $B R C K$, un dispositivo con forma de ladrillo que hace las veces de wifi y router $3 G$ que supera muchos de estos obstáculos (BBC, 2014).

\subsection{Algunos hitos de Ushahidi}

El hecho de haber sido creada para generar informes en un mapa sobre la violencia en Kenia ha influido en los usos posteriores más frecuentes:

$$
\begin{aligned}
& \text { - gestión de información en situaciones de crisis humanita- } \\
& \text { rias; } \\
& \text { - vigilancia del poder; } \\
& \text { - denuncia de problemas sociales y medioambientales; } \\
& \text { - proyectos en defensa de los derechos humanos; } \\
& \text { - curación de contenidos locales. }
\end{aligned}
$$

El hito más relevante de la historia de la plataforma fue la cobertura desplegada a raíz del terremoto que azotó Haití, según Anahi Ayala (Espiritusanto; Gonzalo-Rodríguez, 2011, p. 99). Ushahidi-Haití se activó dos horas después del primer temblor importante, en enero de 2010. Se lanzó el número corto para mensajes SMS 4636 en Haití, permitiendo el envío de información casi en tiempo real y ofreció ayuda con localización mediante mensajes SMS. Se movilizó a más de 300 estudiantes universitarios que buscaron información sobre la crisis de Haití en internet. Otros miles de voluntarios haitianos tradujeron los mensajes cortos y otras informaciones, del criollo al inglés, además de determinar localizaciones específicas.

El 19 de enero de 2010, Ushahidi-Haití se convirtió en una fuente imprescindible de información en tiempo real y geolocalizada, que utilizaban los guardacostas de Estados Unidos, la Cruz Roja, Naciones Unidas, el Departamento de Estado de Estados Unidos, los cuerpos médicos internacionales, y otras organizaciones de primera respuesta (Ayalalacucci, 2011). Al final del proyecto, en agosto de 2010, los voluntarios habían mapeado manualmente más de 3.500 informaciones y habían ayudado a salvar la vida de más de 100 personas. El éxito de la experiencia en Haití permitió una actuación aún más rápida en el terremoto de Chile un mes más tarde y su uso ha demostrado su especial utilidad en la cobertura de otros muchos tipos de desastres naturales como los medioambientales: el vertido de petróleo en el Golfo de México o los frecuentes accidentes de las refinerías de Louisiana (figura 1).

The New York times (Gigidharadas, 2010) destacó en 2010 cómo este nuevo paradigma ha revolucionado la gestión de crisis humanitarias con respecto a la forma en que se venía haciendo hasta el momento. Ushahidi ha mostrado cómo los propios afectados pueden suministrar datos y organizarse para colaborar en las labores de socorro. Representa una nueva frontera de la innovación, donde el espíritu de empresa nace de las dificultades y se centra en hacer más con menos.

El empleo de medios tecnológicos para la vigilancia de los procesos electorales por parte de los ciudadanos ha ido en aumento en los últimos años. Las elecciones de 2009 en Irán marcaron un precedente en la denuncia de posibles fraudes electorales, que llevaron posteriormente a las revueltas ciudadanas. Gau- 
rav Mishra colaboró en Vote report India para un seguimiento en tiempo real de unas largas y complejas elecciones generales en India, que fueron las primeras en utilizar esta aplicación, según cuenta en una entrevista realizada por el equipo de Periodismociudadano.com (periodismociudadano.com, 2015). Hasta entonces sólo se había empleado en situaciones de conflicto armado, crisis o emergencias como las que se vivieron en Gaza o Kenia y esa experiencia sirvió para ampliar la visión.

Además de consolidarse como vigilante del poder en momentos críticos como los procesos electorales, se considera clave poder dar sentido a la información sobre defensa de los derechos humanos. Todo ello mediante agregación, etiquetado, filtrado y geolocalización de la información suministrada por los ciudadanos, los medios de comunicación y los gestores de los distintos proyectos.

Desde 2011 está en constante actualización Syria tracker, un proyecto de Humanitarian Tracker sobre abusos y ataques que sufre el pueblo sirio a diario. Es el mapa más activo de la plataforma con 5.614 reportes recibidos hasta la fecha (figura 2). Para tratar de visibilizar y denunciar la problemática de la violencia contra las mujeres en este país Women's Media Center mantiene vivo el crowdmap Women under siege (figura 3).

Ushahidi también se ha utilizado en campañas de movilización social como Yo digo aquí estoy, creada con el fin de erradicar el trabajo infantil por la Fundación Telefónica en Colombia. 1.800 .000 niños y niñas trabajan en este país y más del $90 \%$ no está identificado. Con este proyecto se les ubica y se les da la oportunidad de tener un futuro mejor.

Otro proyecto fue el de los estudiantes chilenos que en 2013 paralizaron el curso escolar para reclamar la transformación de diversas leyes y por una educación superior gratuita. Chile, país pionero del periodismo ciudadano en América Latina con Atina Chile, se movilizó para reflejar en la Web todos los movimientos sociales que surgieron en torno a la educación y se creó una plataforma de información georeferenciada llamada Chile Movilizado.

Los usos de Ushahidi en un ámbito geográfico más reducido, local o hiperlocal son numerosos (Ushahidi, 2014). Entre otros muchos ejemplos de curación de contenidos locales destaca el realizado en España a través de la creación de un crowdmap de Ushahidi durante la huelga general del 29 de septiembre de 2011. Los ciudadanos utilizaron este mapa para conseguir información en tiempo real sobre necesidades básicas -servicios mínimos, transportes, participación-, ofreciendo repuestas útiles a los usuarios a gran velocidad.
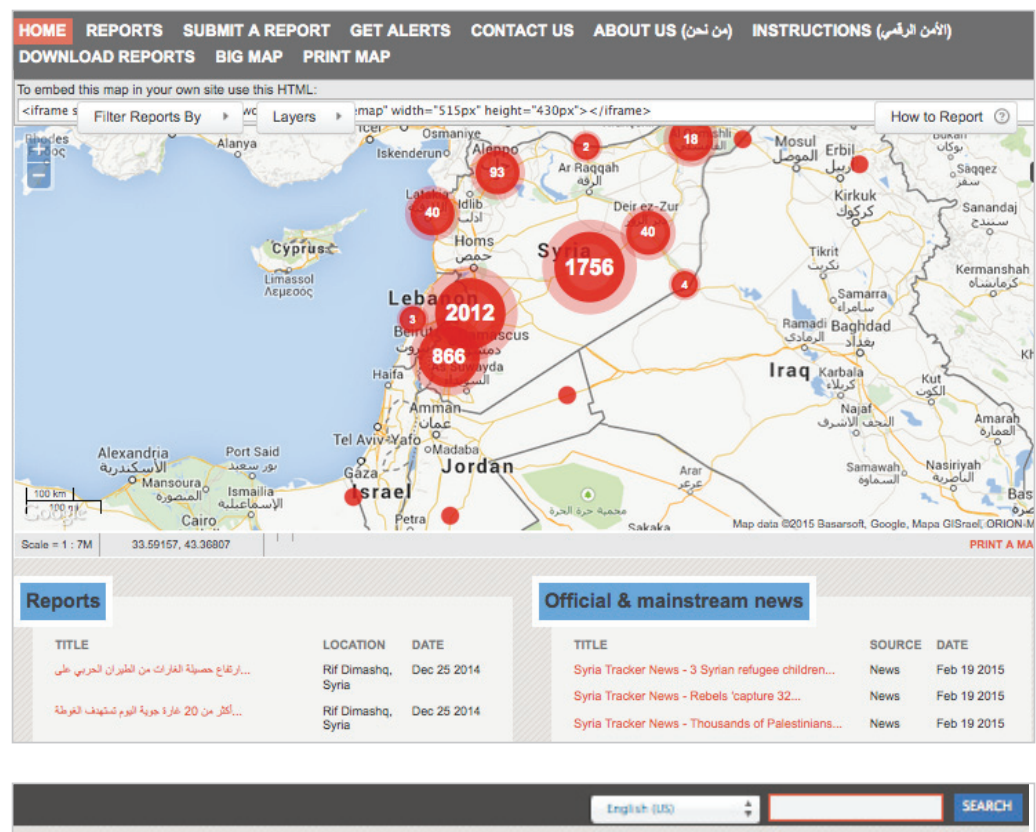

WOMEN'S MEDIA CENTER'S WOMEN UNDER SIEGE

Documenting sexualized violence in Syria

توثيق الاعتداءات الجنسية في سورية

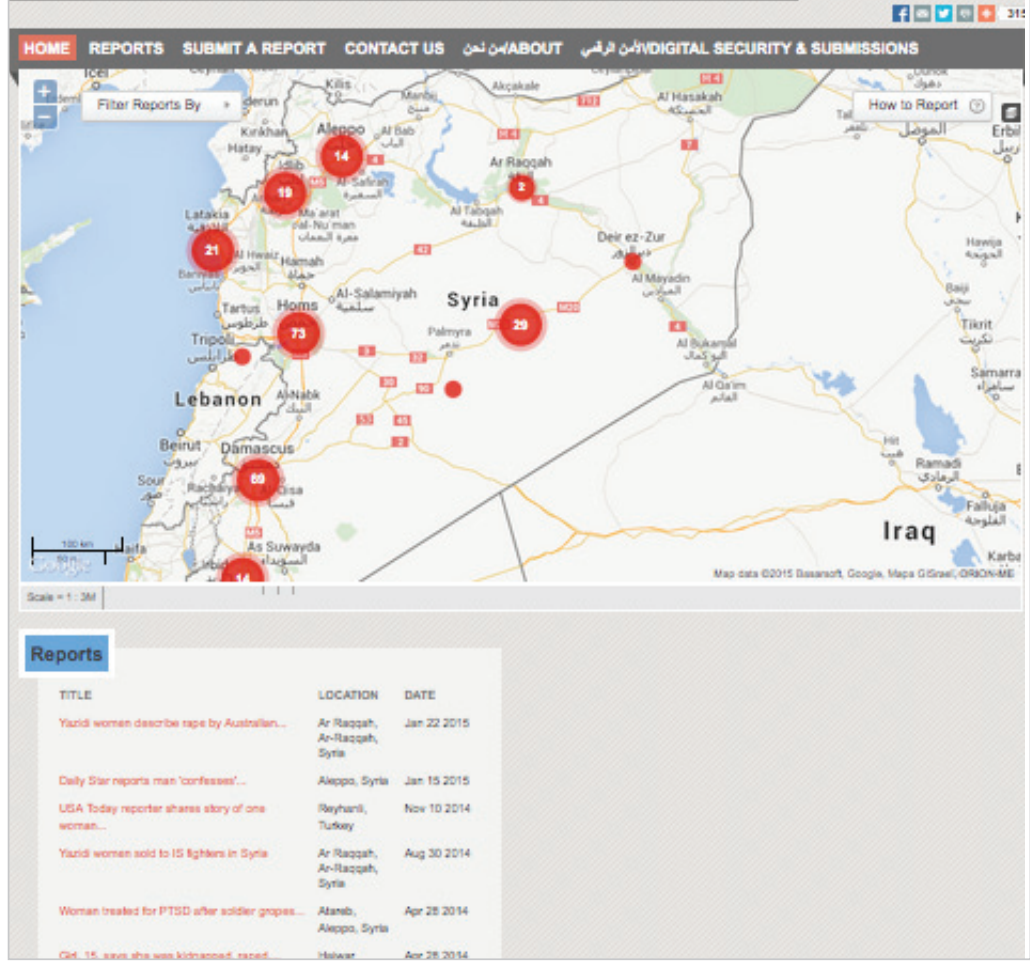

Figuras 2 y 3 . Syria tracker y Women under siege.

https://syriatracker.crowdmap.com

http://www.humanitariantracker.org/\#!syria-tracker/cj00

\section{Ushahidi y periodismo. Resultados y discusión}

Los atentados contra la libertad de expresión no son exclusivos de países con regímenes opresivos. En Europa también se producen numerosas situaciones en las que los periodistas y los medios sufren actos de censura y limitaciones. Media freedom es un proyecto del Index on Censorship y el Osservatorio Balcani e Caucaso para mapear el estado 


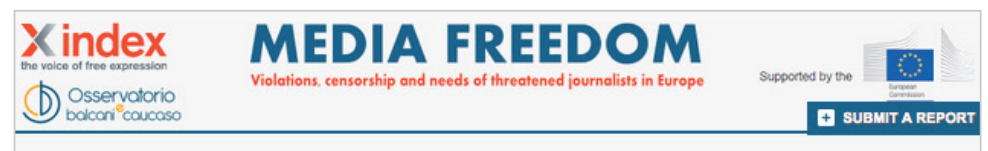

HOME REPORTS SUBMIT A REPORT GET ALERTS CONTACT US LEGAL SUPPORT

DOWNLOAD REPORTS

Index on Censorship and Osservatorio Balcanie Caucaso are joining forces to map the state of media freedom in Europe. With your participation, we are mapping the violations, threats and limitations that European media professionals, bloggers and citizen
journalists face everyday. We are also collecting feedback on what would support journalists in such situations. This mapping project covers European Union member states and candidates for entry. Help protect media freedom and democracy by contributing to this FLIERS - WM NEW PICTURES vDED
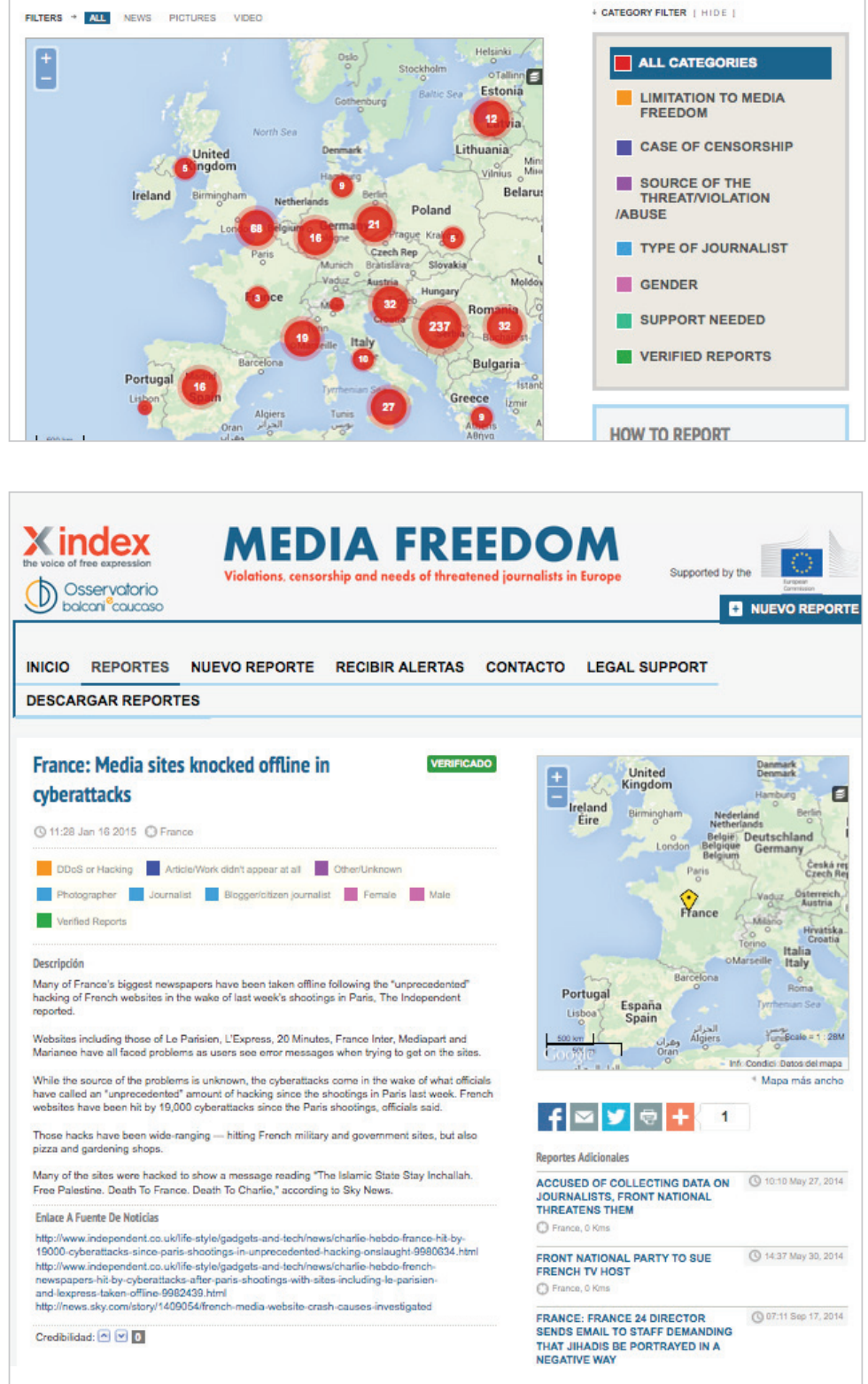

Figuras 4 y 5 . Media Freedom.

http://mediafreedom.ushahidi.com

de la libertad de expresión en Europa. No sólo incluye los ataques a periodistas, sino también a bloggers y periodistas ciudadanos día a día, así como a los propios medios de comunicación, como los ciberataques islamistas a las webs de varios medios franceses tras los asesinatos de París, en enero de 2015, de miembros de la redacción del Charlie Hebdo (figuras 4 y 5 ).
De la investigación realizada sobre la implementación de esta herramienta en las redacciones de los medios se observa que la generación de información en mapas entre periodistas ciudadanos y periodistas es aún incipiente. No obstante, algunos medios referentes ya han experimentado con ella, incluso más de una vez (tabla 1).

La $B B C$ la empleó en varias ocasiones para cubrir una huelga en el metro de Londres. Se pretendía proporcionar un servicio útil a los ciudadanos, con información generada por ellos pero verificada desde la $B B C$ antes de publicarla (Wardle, 2010). El 2 de noviembre de 2011 se publicaron 55 reportes y se desconoce el número de colaboraciones en otro mapa del mismo tema del 7 de septiembre de 2010 (Oliver, 2010).

The Huffington post ha utilizado Crowdmap de Ushahidi para recibir información de los ciudadanos durante el huracán Sandy (81 reportes) (figura 6). En la web de Ushahidi se presenta a The Huffington post como partner en proyectos de colaboración. Esta relación se inició a partir del interés del diario en 2012 "de cubrir la recuperación económica de Estados Unidos de la gran recesión de 2008" (Ushahidi, 2015b). Sin embargo, este crowdmap en concreto no ha dejado ninguna huella en internet.

Ushahidi y Al jazeera también colaboraron en 2009 para poner en marcha War on Gaza (Qdev, 2010), un sitio dedicado al conflicto entre israelíes y palestinos que tiene ese territorio como escenario principal. No obstante, tampoco se ha podido encontrar este proyecto. Posteriormente, se unieron de nuevo para Somalia speaks, que recibió 4.000 reportes. Según declaraciones de Soud Hyder de Al jazeera en la PBS (Ulbricht, 2012): "Queríamos conocer la perspectiva de los ciudadanos somalíes y que nos dijeran cómo la crisis ha afectado a sus vidas y a la diáspora somalí".

En la wiki de Ushahidi se menciona un proyecto similar de Al jazeera titulado Uganda speaks (Usahidi, 2012), aunque este crowdmap no se ha podido encontrar.

La visita del Papa Benedicto XVI al Reino Unido sirvió para que The guardian creara un crowdmap (Papal visit) que combinaba informes verificados por el diario (85 reportes) y otros medios con información valiosa de quienes oían, escribían, fotografiaban o grababan el acontecimiento (Lewis, 2010). El subtítulo del proyecto era "Sigue al Papa en el Reino Unido" y en la presentación se advertía de las rigurosas condiciones de publicación (material relevante, no ofensivo, contrastable).

El segundo experimento de The guardian se enmarcó dentro de una investigación sobre el impacto de los recortes en 
Tabla 1. Implementación de Ushahidi en medios

\begin{tabular}{|c|c|c|c|}
\hline Medio & Período & Evento & $N^{\circ}$ reportes \\
\hline The guardian & $\begin{array}{l}15 / 09 / 2010 a \\
19 / 09 / 2010\end{array}$ & $\begin{array}{l}\text { Pope Benedict XVI Visit UK } \\
\text { https://papalvisit.crowdmap.com }\end{array}$ & 85 \\
\hline The guardian & Octubre 2010 & $\begin{array}{l}\text { Leeds cuts wacht } \\
\text { (no está accesible en la actualidad) }\end{array}$ & 84 \\
\hline$B B C$ & $\begin{array}{l}03 / 11 / 2010 a \\
3 / 11 / 2010\end{array}$ & $\begin{array}{l}\text { London tube strike map - November } 28^{\text {th }} / 29^{\text {th }} 2010 \\
\text { https://tubestrike.crowdmap.com }\end{array}$ & 55 \\
\hline The Huffington post & $\begin{array}{l}28 / 10 / 2012 a \\
1 / 11 / 2012\end{array}$ & $\begin{array}{l}\text { Huffington Post Sandy stormwatch } \\
\text { https://hpsandy.crowdmap.com }\end{array}$ & 81 \\
\hline Al jazeera & $12 / 2011$ & $\begin{array}{l}\text { Somalia speaks } \\
\text { (No accesible en la actualidad) }\end{array}$ & 4.000 \\
\hline Aljazeera & 2009 & $\begin{array}{l}\text { War on Gaza } \\
\text { (No accesible en la actualidad) }\end{array}$ & Desconocido \\
\hline Aljazeera & Desconocido & $\begin{array}{l}\text { Uganda speaks } \\
\text { (No accesible en la actualidad) }\end{array}$ & Desconocido \\
\hline Watershed post & $\begin{array}{c}28 / 10 / 2012 a \\
5 / 11 / 2012\end{array}$ & $\begin{array}{l}\text { Sandy stormwatch } \\
\text { https://catskillshurricanesandy.crowdmap.com }\end{array}$ & 228 \\
\hline Houston chronicle & $\begin{array}{l}7 / 12 / 2011 \text { a } \\
7 / 12 / 2011\end{array}$ & $\begin{array}{l}\text { The Christmas lightsmap } \\
\text { https://chronchristmaslights.crowdmap.com/feeds?l=dv }\end{array}$ & 73 \\
\hline Houston chronicle & $\begin{array}{l}13 / 1 / 11 \text { a } 4 \\
/ 4 / 2011\end{array}$ & $\begin{array}{l}\text { Houston pothole patrols } \\
\text { https://badhoustonstreets.crowdmap.com/reports }\end{array}$ & 651 \\
\hline Houston chronicle & Desconocido & $\begin{array}{l}\text { Houston storm reports } \\
\text { No presenta ninguna entrada en la actualidad } \\
\text { https://houweather.crowdmap.com/main?l=es_VE\&I=en_GB }\end{array}$ & Desconocido \\
\hline $\begin{array}{l}\text { WNYC } \\
\text { New York public radio }\end{array}$ & $5 / 2013$ & $\begin{array}{l}\text { Cicada tracker } \\
\text { http://project.wnyc.org/cicadas }\end{array}$ & $\begin{array}{c}\text { Numerosas. No figura la } \\
\text { cantidad precisa }\end{array}$ \\
\hline Hamilton reporter & $\begin{array}{l}16 / 3 / 2012 a \\
26 / 9 / 2012\end{array}$ & $\begin{array}{l}\text { Hamilton reporter } \\
\text { https://hamiltonreporter.crowdmap.com }\end{array}$ & 120 \\
\hline
\end{tabular}

el sector público en la ciudad de Leeds (Leeds cuts watch). Mediante crowdmapping se pretendió conocer cómo estos recortes afectaban a las personas de forma individual y a las comunidades. Además de crear una imagen única de donde están perjudicando con mayor intensidad (Baron, 2011). Recibió 84 reportes (figura 7).

El equipo del medio se encargó de garantizar que sólo los informes revisados obtuvieran una etiqueta de "verificado". Destaca la perfecta integración entre la herramienta y la web del diario británico, tanto en el diseño como en la usabilidad y la navegabilidad, lo que demuestra la versatilidad de este software.

Algunos medios locales o hiperlocales también han puesto en marcha proyectos en este servicio, todos estadounidenses: Houston chronicle, Watershed post, la emisora de radio pública de Nueva York WNYC, y el portal Hamilton reporter.

En el caso de Houston chronicle, el objetivo tenía un componente participativo y de entretenimiento. Con el proyecto The Christmas lightsmap (Ushahidi, 2012) insertado en la versión digital del diario (Chron.com) se invitó a los ciudadanos a enviar informaciones y material gráfico de lugares iluminados por Navidad. Se enviaron 73 reportes en 10 días. Otras experiencias de este medio son:

- Houston pothole patrol, donde los conductores enviaron 651 reportes de carreteras de Texas en malas condiciones;

- Houston storm reports, que no presenta ninguna entrada en la actualidad.
El diario local Watershed post realizó un crowdmap sobre la incidencia del huracán Sandy en la zona neoyorquina de Catskills en 2012 (Hurricane Sandy coverage) al que denominó Hurricane Sandy live blog (Ushahidi, 2012) y que recibió 228 informaciones de ciudadanos. La utilización de esta aplicación para la retransmisión en vivo de un hecho (a través de reporteros y ciudadanos) es uno de los mayores valores que añade esta plataforma al periodismo.

Se constata la importancia de la participación activa de los usuarios en la generación de contenido en tiempo real

WNYC, con el fin de predecir el resurgimiento de los enjambres de cigarras, solicitó a los residentes de determinadas áreas que usaran sensores para registrar la temperatura del suelo. Las tomas enviadas por los ciudadanos se desplegaron en un mapa en la web de la WNYC (Aitamurto, 2015). En la misma se explicaba cómo hacer un sensor de fabricación casera y los resultados obtenidos se enviaban a un grupo de investigadores científicos que recogían los datos.

Por último, el portal Hamilton reporter de Canadá es un repositorio de reportes enviados por ciudadanos, organizaciones y empresas, y un agregador de noticias de los medios. El portal etiqueta como "verificado" determinadas entradas 
de un total de 120 publicadas.

Teniendo en cuenta la muestra presentada en los párrafos anteriores, se detecta que hasta el momento los medios de comunicación han utilizado Ushahidi para:

- proporcionar información útil (periodismo de servicio);

- conocer la perspectiva de los ciudadanos;

- completar con otros puntos de vista proyectos de investigación;

- dar la oportunidad a los lectores de convertirse en reporteros;

- obtener material visual captado por los ciudadanos sobre un evento de interés para una comunidad;

- detectar una zona concreta con un mayor índice de impacto de un problema.

En este listado de posibilidades o ventajas se echa de menos la humanización de la información. Aunque ello quede implícito en algunos casos, los mapas y los reportes no dejan de ser elementos en una sola dimensión que necesitan ser analizados por periodistas y expertos para comprender el alcance y la envergadura de un problema.

A la vista de las fechas que tienen los proyectos y de lo reducido que resulta el universo de la muestra surge una cuestión que nos parece clave: ¿por qué no se han realizado más proyectos con Crowdmap-Ushahidi? Si nos fijamos en los grandes medios que lo han adoptado, son precisamente los que lideran la participación, la innovación y la adaptación a las nuevas corrientes periodísticas. Dado que la plataforma se basa en el envío de sms, tweets, mensajes de texto y material gráfico o vídeo, la respuesta podría encontrarse extrapolando provisionalmente y con ciertas reservas los resultados de las investigaciones sobre el uso de Twitter por los medios. Como señala López-Meri (2015, pp. 35-38), citando a Said-Hung et al. (2013), "los medios todavía son conservadores respecto al uso de redes sociales", y muchos periodistas siguen mediados por modelos tradicionales ajenos a escenarios digitales. Esta misma autora cita a otras voces críticas:

- García-Avilés, Kaltenbrunner y Meier (2014) consideran "que se debería implementar la interacción con el público para obtener rendimiento económico"

- Carrera-Álvarez et al. (2012) afirman que lo inédito es poco frecuente en Twitter, ya que los periodistas, "más que crear nuevos contenidos, diseminan y revitalizan argumentos preexistentes, sin aprovechar la oportunidad que brinda el crowdsourcing para generar informaciones en colaboración con los usuarios".

Si bien podrían considerarse igualmente como válidos estos argumentos para el caso del crowdmapping, existen otras variables a tener en cuenta y que deberían ser exploradas en estudios posteriores, mediante entrevistas semi-estruc- turadas a responsables y periodistas de medios y freelance:

- dificultades mencionadas en algunos estudios citados para la puesta en práctica de Ushahidi: capacitación en la herramienta, conocimiento de situaciones de crisis, verificación de la información y validación de las imágenes;

- reciente creación de laboratorios de medios que indagan o crean nuevas aplicaciones y prueban nuevas formas de storytelling. En este tema, los infografistas de los medios tienen una amplia trayectoria en la realización de mapas informativos e interactivos y dominan numerosos programas de diseño;

- mayor desarrollo en los últimos años de plataformas de curación y agregación de contenidos de redes sociales, blogs, etc., y de aplicaciones, que nacidas con otra finalidad llevan a cabo proyectos humanitarios con el fin de ayudar para su uso en situaciones de crisis (StreetMapView);

- falta de tiempo y de personal en los medios y falta de externalización de determinados proyectos de cierta complejidad;

- cuenta de resultados negativa: que no sean rentables para las empresas mediáticas cuando el número de visitas a estos proyectos no supera unos mínimos.

\section{Conclusiones}

Se está produciendo una externalización masiva de elementos propios del proceso periodístico mediante la utilización de herramientas de innovación social como Ushahidi, que se integran en la práctica informativa tanto por la ciudadanía como por periodistas. Esta plataforma ha demostrado toda la potencialidad del fenómeno. En el caso del crowdmapping, se comprueba la importancia de las infotecnolo- 
gías y en concreto de los dispositivos móviles.

Se constata:

- importancia de la participación activa de los usuarios en la generación de contenido en tiempo real;

- su valor a la hora de ayudar en casos de crisis y emergencias, como los terremotos en Haití y Chile;

- su valor en los procesos electorales como sistema de vigilancia electoral;

- su valor en la curación de contenidos locales de todo tipo.

Al tratarse de una herramienta basada en software libre y sin necesidad de instalación en servidores propios, es fácil su utilización por medios, instituciones o comunidades de todo tipo, tamaño y condición. Los ciudadanos, con los recursos disponibles a su alcance y salvando los problemas de verificación y contraste, pueden convertirse en fuente informativa directa de los medios de comunicación, aportando valor añadido a la información.

La creación de mapas colaborativos entre periodistas y ciudadanos se muestra como una posibilidad real aplicable a diversas situaciones, en especial, en los casos de crisis humanitarias, catástrofes naturales, accidentes y todas aquellas situaciones de emergencia que requieren de una respuesta informativa rápida, en las que el periodismo cumple su función social de informar sobre lo que los ciudadanos precisan con mayor urgencia.

Ushahidi no puede garantizar al $100 \%$ la seguridad de quienes envían información

A partir de los resultados obtenidos en relación con la utilización de mapas de información con Ushahidi por parte de los medios, se detecta un área temática en la que confluyen los intereses de las comunidades (tanto de ciudadanos como de voluntarios en proyectos de ayuda humanitaria) y los medios; y del interés compartido pueden surgir otros proyectos periodísticos innovadores que utilicen las tecnologías de la geolocalización de información en tiempo real.

Ushahidi constituye una plataforma donde los ciudadanos pueden verificar las informaciones ofrecidas por medios de comunicación (a veces de regímenes dictatoriales o con una dudosa democracia, y con una libertad de expresión cercenada), y viceversa, y donde la utilización parcial de información puede ser fácilmente detectada por ambos, los medios y los ciudadanos. Al mismo tiempo éstos pueden utilizarla para recopilar información sobre eventos concretos ayudados por la masa generadora de contenido. Tan sólo han experimentado con la herramienta para interactuar con sus lectores algunos grandes medios de comunicación internacionales y algunos medios locales.

\section{Los medios no siempre mantienen en el tiempo y en abierto las obras realizadas con Ushahidi}

Aunque Ushahidi ha pasado de ser un mapa en el que se georreferenciaba la crisis electoral vivida en Kenia en 2008 a una red social de información generada por una masa implicada en un mismo evento o concepto, necesita ser adaptada al entorno y la situación concreta. Por otro lado, existe una dificultad para verificar los datos en tiempo real, aunque esta función mejora con cada versión de Swiftriver. Más grave es la imposibilidad de asegurar el anonimato del envío de información cuando puede correr peligro la integridad física o psíquica de las personas que colaboran enviando esa información. En el caso de la cobertura de violaciones de los derechos humanos o de conflictos en regímenes represivos, la plataforma Ushahidi no puede garantizar al $100 \%$ la seguridad de quienes envían información.

Por último, cabe señalar las limitaciones de esta investigación derivadas de la pérdida de material digital en internet, debido a que los medios no siempre mantienen en el tiempo y en abierto las obras realizadas con Ushahidi, lo que imposibilita los análisis de algunos proyectos. También sería de interés entrevistar a los autores de estos proyectos periodísticos, sobre todo cuando no han narrado previamente esta experiencia por otras vías, como hiciera uno de los autores de la $B B C$ en su blog personal. 


\section{Notas}

1. Esta investigación forma parte del proyecto Big data, redes sociales y periodismo de datos: aplicación de las herramientas de monitorización al análisis de fuentes y contenidos periodísticos, financiado por el Ministerio de Economía y Competitividad dentro del Plan Nacional Proyectos de I $+D+l$, del Programa Estatal de Investigación, Desarrollo e Innovación orientada a los Retos de la Sociedad. Referencia: CSO2013-47767-C2-1-R. Asimismo, se enmarca dentro de las acciones desarrolladas por el grupo de investigación Paseet de la Universidad Carlos III de Madrid.

2. Entre otros autores citados en el epígrafe 4: "Ventajas y desventajas del uso de mapas de crisis".

3. No se han señalado diferencias entre estos términos, porque no dejan de ser palabras similares de un mismo tipo de periodismo.

\section{Bibliografía}

Aitamurto, Tanja (2011). "The impact of crowdfunding on journalism: Case study of Spot.us, a platform for community-funded reporting". Journalism practice, v. 5, n. 4, pp. 429-445.

http://dx.doi.org/10.1080/17512786.2010.551018

Aitamurto, Tanja (2015). "Motivation factors in crowdsourced journalism: social impact, social change and peer learning". International journal of communication, v. 9, pp. 3523-3543.

http://ijoc.org/index.php/ijoc/article/view/3481

Aitamurto, Tanja (2016). "Crowdsourcing as a knowledgesearch method in digital journalism: Ruptured ideals and blended responsibility". Digital journalism, v. 4, n. 2, pp. 280-297.

http://goo.gl/UCr1xO

http://dx.doi.org/10.1080/21670811.2015.1034807

Allan, Stuart (2013). Citizen witnessing: Revisioning journalism in times of crisis. Cambridge, UK: Polity Press. ISBN: 978 0745651958

Ayala-lacucci, Anahi (2011). "Periodismo ciudadano: geolocalización”. En: Espiritusanto, Óscar; Gonzalo-Rodríguez, Paula. Periodismo ciudadano. Evolución positiva de la comunicación. Barcelona/Madrid: Ariel/Fundación Telefónica, pp. 100-102. ISBN: 9788408104162

http://www.revistacomunicar.com/pdf/2011-12-15-libroperiodismo-ciudadano.pdf

Bailard, Catie-Snow; Livingston, Steven (2014). "Crowdsourcing accountability in a Nigerian election". Journal of information technology \& politics, v. 11, n. 4, pp. 349-367. http://dx.doi.org/10.1080/19331681.2014.947056

Baron, John (2011). "Latest cutswatch reporst highlight cuts to services in Leeds". The guardian, 16 March.

http://www.theguardian.com/leeds/2011/mar/16/leedscutswatch-latest-cuts

BBC (2014). “Ushahidi's BRCK Released”. Click, 6 August. http://www.bbc.co.uk/programmes/p023sp0k

Beltrán, Gersón (2016). Geolocalización online. La impor- tancia del dónde. Barcelona: Editorial UOC, colección El profesional de la información, n. 32. ISBN: 9788491161592

Brabham, Daren C. (2008). "Crowdsourcing as a model for problem solving: An introduction and cases". Convergence, v. 14, n. 1, pp. $75-90$.

http://goo.gl/7UYDh

http://dx.doi.org/10.1177/1354856507084420

Brabham, Daren C. (2013). Crowdsourcing. Cambridge, MA: MIT Press. ISBN: 9780262518475

http://wtf.tw/ref/brabham.pdf

Bradshaw, Paul; Brightwell, Andy (2012). "Crowdsourcing investigative journalism: Help me investigate - A case study". En: Siapera, Eugenia; Veglis, Andreas (eds.). The handbook of global online journalism, pp. 253-271) Oxford, UK: Wiley-Blackwell. ISBN: 9781118313954

Brandusescu, Ana; Sieber, Renée E.; Jochems, Sylvie (2015). "Confronting the hype: The use of crisis mapping for community development". Convergence, online, May 18, pp. 1-17.

http://dx.doi.org/10.1177/1354856515584320

Carrera-Álvarez, Pilar; Sainz-de-Baranda-Andújar, Clara; Herrero-Curiel, Eva; Limón-Serrano, Nieves (2012). “Journalism and social media: How Spanish journalists are using Twitter". Estudios sobre el mensaje periodístico, n. 18, pp. 31-53.

http://dx.doi.org/10.5209/rev_eSMP.2012.v18.n1.39353

Cohen, Sarah; Hamilton, James T.; Turner, Fred (2011). "Computational journalism". Communications of the ACM, Oct., v. 54, n. 10, pp. 66-71.

http://cacm.acm.org/magazines/2011/10/131400computational-journalism/fulltext http://dx.doi.org/10.1145/2001269.2001288

Chamales, George; Baker, Rob (2011). "Securing crisis maps in conflict zones". En: 2011 IEE Global humanitarian technology conf., Seattle, WA, pp. 426-430.

http://dx.doi.org/10.1109/GHTC.2011.47

Dailey, Dharma; Starbird, Kate (2014). “Journalists as crowdsourcerers: Responding to crisis by reporting with a crowd". Computer supported cooperative work archive, v. 23, n. 4-6, pp. 445-481.

http://dx.doi.org/10.1007/s10606-014-9208-z

Daniel, Anna; Flew, Terry (2010). "The guardian reportage of the UK MP expenses scandal: A case study of computational journalism". En: The record of the communications policy and research forum, 15-16 Nov., Sydney, pp. 186-194. Sydney: Network Insight Institute.

http://eprints.qut.edu.au/38701/2/38701.pdf

Davidi, Adam; Berliner, Michael (2014). "How the web is amplifying citizens' voices: Q\&A with Ory Okolloh". The guardian, 29 May.

http://www.theguardian.com/media-network/medianetwork-blog/2014/may/29/citizen-activism-internet-africaomidyar

Diezhandino-Nieto, María-Pilar (1994). Periodismo de servicio. Barcelona: Bosch. ISBN: 9788476762936 
Diezhandino-Nieto, María-Pilar (dir.) (2007). Periodismo en la era de internet. Madrid: Ariel/Fundación Telefónica. http://goo.gl/n88FD8

Domingo, David; Quandt, Thorsten; Heinonen, Ari; Paulussen, Steve; Singer, Jane B; Vujnovic, Marina (2008). "Participatory journalism practices in the media and beyond". Journalism practice, v. 2, n. 3, pp. 326-342.

http://jclass.umd.edu/classes/jour698m/domingo.pdf

http://dx.doi.org/10.1080/17512780802281065

Espiritusanto, Óscar; Gonzalo-Rodríguez, Paula (2011). Periodismo ciudadano. Evolución positiva de la comunicación. Barcelona/Madrid: Ariel/Fundación Telefónica. ISBN: 97884 08104162

http://www.revistacomunicar.com/pdf/2011-12-15-libroperiodismo-ciudadano.pdf

Estellés-Arolas, Enrique; González-Ladrón-de-Guevara, Fernando (2012a). "Towards an integrated crowdsourcing definition". Journal of information science, v. 38, n. 2, pp. 189-200.

http://dx.doi.org/10.1177/0165551512437638

Estellés-Arolas, Enrique; González-Ladrón-De-Guevara, Fernando (2012b). "Tasks-based classification of crowdsourcing initiatives". El profesional de la información, v. 21, n. 3, pp. 283-291.

http://dx.doi.org/10.3145/epi.2012.may.09

Fumero-Reverón, Antonio (2011). “IRC 2.0. Medios para la información, la relación y la comunicación en la web 2.0”. El profesional de la información, v. 20, n. 6, pp. 605-609.

http://recyt.fecyt.es/index.php/EPI/article/view/epi.2011. nov.01

http://dx.doi.org/10.3145/epi.2011.nov.01

Furtado, Vasco; Caminha, Carlos; Ayres, Leonardo; Santos, Henrique (2012). "Open government and citizen participation in law enforcement via crowd mapping". Intelligent systems, IEEE, v. 27, n. 4, pp. 63-69.

http://dx.doi.org/10.1109/MIS.2012.80

García-Avilés, José-Antonio; Kaltenbrunner, Andy; Meier, Klaus (2014). "Media convergence revisited". Practice journalism, v. 8, n. 5, pp. 573-584.

http://dx.doi.org/10.1080/17512786.2014.885678

García-De-Torres, Elvira (2010). “Contenido generado por el usuario: aproximación al estado de la cuestión". El profesional de la información", v. 19, n. 6, pp. 585-594.

http://recyt.fecyt.es/index.php/EPI/article/view/epi.2010. nov.04

http://dx.doi.org/10.3145/epi.2010.nov.04

Gigidharadas, Anand (2010). "Africa's gift to Silicon Valley: How to track a crisis". The New York times, 13 March. http://www.nytimes.com/2010/03/14/weekinreview/14giridharadas. $h t m l$ ? $r=0$

Gillmor, Dan (2004). We the media: Grassroots journalism by the people, for the people. O'Reilly Media. ISBN: 9780 596102272

Gonzalo-Rodríguez, Paula (2011). "Hitos del periodismo ciudadano: del tsunami de Indonesia a las revueltas de Tú- nez". En: Espiritusanto, Óscar; Gonzalo-Rodríguez, Paula. Periodismo ciudadano. Evolución positiva de la comunicación. Barcelona: Ariel, pp.29-50. ISBN: 9788408104162 http://www.revistacomunicar.com/pdf/2011-12-15-libroperiodismo-ciudadano.pdf

Gonzalo-Rodríguez, Paula (2016). “Participación ciudadana y gestión de crisis, 5 organizaciones que debes conocer". Periodismociudadano.com, 10 febrero.

http://www.periodismociudadano.com/2016/02/10/ participacion-ciudadana-y-gestion-de-crisis-5-organizacionesque-debes-conocer

Greengard, Samuel (2011). "Following the crowd". Communication of the ACM, v. 54, n. 2, pp. 20-22.

http://dx.doi.org/10.1145/1897816.1897824

Grove, Nicole S. (2015). "The cartographic ambiguities of HarassMap.com: Crowdmapping security and sexual violence in Egypt". Security dialogue, v. 46, n. 4, pp. 345-364. http://socialsciences.people.hawaii.edu/publications_lib/ Grove\%20Cartographic\%20Ambiguities\%20SD.pdf http://dx.doi.org/10.1177/0967010615583039

Hänska-Ahy, Maximillian; Shapour, Roxanna (2013). “Who's reporting the protests? Converging practices of citizen journalists and two BBC world service newsrooms, from Iran's election protests to the Arab uprisings". Journalism studies, v. 14, n. 1, pp. 29-45.

http://eprints./se.ac.uk/41674

http://dx.doi.org/10.1080/1461670X.2012.657908

Holton, Avery E.; Coddington, Mark; Gil-de-Zúñiga, Homero (2013). "Whose values? Citizen journalism and journalistic values through the lens of content creators and consumers". Journalism practice, v. 7, n. 6, pp. 720-737.

https://goo.gl/2JrEzW

http://dx.doi.org/10.1080/17512786.2013.766062

Howe, Jeff (2006). "The rise of crowdsourcing". Wired, 1 June.

http://www.wired.com/wired/archive/14.06/crowds.html

Howe, Jeff (2009). Crowdsourcing: Why the power of the crowd is driving the future of business. Crown Business. ISBN: 9780307396211

Internews Center for Innovating \& Learning (2012). Mapping the maps. Washington DC.

https://irevolution.files.wordpress.com/2013/01/ internewswpcrowdglobe_web-1.pdf

Lévy, Pierre (2004). Inteligencia colectiva: por una antropología del ciberespacio. Organización Panamericana de Salud. http://inteligenciacolectiva.bvsalud.org/public/documents/ pdf/es/inteligenciaColectiva.pdf

Lewis, Paul (2010). "Pope visit: help us to crowdmap every detail". The guardian, 16 Sept.

http://www.theguardian.com/world/blog/2010/sep/16/ pope-visit-crowdmap

López-Guillén, Karla-Ivon; Flores-Mendoza, Uriel; WeltiSantos, Larissa (2011). "Crowdmap and Ushahidi: to obtain and visualize traffic congestion information in Mexico City". En: Procs. of the $4^{\text {th }}$ ACM SIG Spatial international workshop 
on computational transportation science (CTS'11). ACM New York, pp. 24-27,

http://dx.doi.org/10.1145/2068984.2068989

López-Meri, Amparo (2015). "El uso de Twitter en el periodismo: un estado de la cuestión". Rae-ic, v. 2, n. 4, pp. 34-41. http://www.novosmedios.org/revista/index.php/AEICp/ article/view/96

López-Pan, Fernando (2010). "La periodística y sus partes. La propuesta de Josep-Maria Casasús". Sphera pública, n. 10, pp. 237-258.

http://www.redalyc.org/pdf/297/29719345016.pdf

Meier, Patrick (2012). "Crisis mapping in action: How open source software and global volunteer networks are changing the world, one map at a time". Journal of map \& geography libraries, v. 8, n. 2, pp. 89-100.

http://dx.doi.org/10.1080/15420353.2012.663739

Mortensen, Tara; Keshelashvili, Ana; Weir, Tom (2016). "Who we are: A Q-study of types of citizen journalists". Digital journalism, v. 4, n. 3.

http://dx.doi.org/10.1080/21670811.2015.1053506

Oliver, Laura (2010). “BBC using Ushahidi's crowdmap to plot tube strikes". Journalism.co.uk, 7 Sept.

https://www.journalism.co.uk/news/bbc-using-ushahidi039-s-crowdmap-to-plot-tube-strikes/s2/a540447

Onuoha, Mimi; Pinder, Jeanne; Schaffer, Jan (2015). Guide to crowdsourcing. Tow Center for Digital Journalism, Columbia Journalism School, Columbia University, NY.

http://towcenter.org/research/guide-to-crowdsourcing/\#verificationand-legal-issues

Palmer, Lindsay (2014). "Ushahidi at the Google interface: critiquing the "geospatial visualization of testimony'". Continuum: Journal of media \& cultural studies, v. 28, n. 3, pp. 342-356.

http://dx.doi.org/10.1080/10304312.2014.893989

Periodismociudadano.com (2010). "Clay Shirky destaca la importancia de la innovación en el periodismo ciudadano hiperlocal". Periodismociudadano.com, 7 abril.

https://www.youtube.com/watch?v=362pou0TVvg

Periodismociudadano.com (2015). "Gaurav Mishra y Vote report India: Información geolocalizada en tiempo real". Periodismociudadano.com, 12 mayo.

https://www.youtube.com/watch?v=s5C21XUdljE

Plaza, Analía (2014). "Ayudar tras la pantalla es posible: lo que aprendimos con el tifón de Filipinas y OpenStreetMap". Eldiario.es, 30 enero. http://goo.gl/rsrOo2

Qdev (2010). "Site of note: Ushahidi". Gotta quirk, 6 September. http://www.gottaquirk.com/2010/09/06/site-of-noteushahidi-2

Quinn, Alexander J.; Benjamin B. Bederson (2011). “Human computation: a survey and taxonomy of a growing field". En: Proceedings of the SIGCHI Conference on human factors in computing systems, May 7-12, Vancouver, Canada. http://www.cs.umd.edu/ bederson/images/pubs_pdfs/ p1403-quinn.pdf

Rheingold, Howard (2004). Multitudes inteligentes: la próxima revolución social. Gedisa. ISBN: 8497840623

Rheingold, Howard (2011). "Prólogo". En: Espiritusanto, Óscar; Gonzalo-Rodríguez, Paula. Periodismo ciudadano. Evolución positiva de la comunicación. Barcelona/Madrid: Ariel/ Fundación Telefónica, pp. VII-VIII. ISBN: 9788408104162

Said-Hung, Elías; Serrano-Tellería, Ana; García-de-Torres, Elvira; Yerezers'ka, Lyudmyla; Calderín, Mabel (2013). "La gestión de los social media en los medios informativos iberoamericanos". Comunicación y sociedad, v. 26, n. 1, pp. 67-92. http://www.unav.es/fcom/communication-society/es/ resumen.php?art_id $=437$

Shirky, Clay (2008). Here comes everybody: The power of organizing without organizations. Penguin Press. ISBN: 978 0143114949

Singer, Jane B.; Hermida, Alfred; Domingo, David; Heinonen, Ari; Paulussen, Steve; Quandt, Thorsten; Reich, Zvi; Vujnovic, Marina (2011). Participatory journalism: Guarding open gates at online newspapers. NY: Wiley-Blackwell. ISBN: 9781444332261

Ulbricht, Melissa (2012). "Ushahidi join in project to connect Somalia diaspora via SMS". Al jazeera, 6 Jan.

http://www.pbs.org/idealab/2012/01/al-jazeera-ushahidijoin-in-project-to-connect-somalia-diaspora-via-sms003.html

Ushahidi (2012). "Citizen media”. Community wiki, 18 April. https://wiki.ushahidi.com/display/WIKI/Citizen+Media

Ushahidi (2014). "Types of maps". Community wiki, 9 April. https://wiki.ushahidi.com/display/WIKI/Types+of+Maps

Ushahidi (2015a). "Status of the board of directors". Ushahidi blog, 21 Nov.

https://www.ushahidi.com/blog/2015/11/21/ushahidistatus-of-the-board-of-directors

Ushahidi (2015b). "Huffington post first-hand". Ushahidi. https://www.ushahidi.com/case-studies/huffington-postfirst-hand

Wardle, Claire (2010). "The day after. Lessons learned from the crowdmap experience". Clairewardle.com http://clairewardle.com/2010/09/08/the-day-after$\%$ E2\%80\%93-lessons-learned-from-the-crowdmap-experience 